\title{
Cargo Cult Science
}

\author{
MICHAEL HANLON \\ London, UK. E-mail: mikehanlon1964@gmail.com
}

\begin{abstract}
There is plenty of stuff out there that looks like science, sounds like science and yet which is no more science than the 'cargo cult' aircraft and landing strips constructed by Pacific Islanders in the 1940s and 1950s were functional technology. My talk is not so much about the usual suspects - homeopathy, crystal healing, UFOs and the like - but other areas of cargo-cult science that sit far closer to the high altar of respectability. We take far too much for granted in science, and this can be seen in the replicability (or otherwise) of peer-reviewed studies, the phenomenon of publication bias, the so-called Decline Effect and the persistence of folk myths such as the one that describes how aeroplanes fly.
\end{abstract}

The late American physicist Richard Feynman was one of the finest science communicators in history. He lived in an age before soundbites and before the rising tide of science popularisation became a flood. How we need someone like him now.

The title of this paper is borrowed from the theme of a historic address to students given by Feynman in 1974 at Caltech in Pasadena. In his talk Feynman pointed out that in the Middle Ages there were all sorts of strange ideas around, such as the one which said that eating the horn of rhinoceroses increased potency.

In fact, the question, 'Does eating rhino horn make me better in bed' is a scientific one, in that it can (at least in theory) be answered empirically. We can imagine the experiment, the control group, the ethics board and so on. The question is: why, despite the fact that we have an answer and we have had one for some time, are we down to the last few rhinos?

The aphrodisiac effects of rhino horns are obvious junk science but sometimes cargo cult science wears some very respectable clothes indeed. The example given by Feynman concerned the famous oil-drop experiment conducted by Robert Millikan in 1909 to measure the electrical charge of an electron. The experiment involved the use of an electrical field to counteract the effect of gravity on a tiny droplet of oil that was electrically charged. Millikan found that by using droplets of different (known) sizes he was able to calculate that the electrical charges needed to keep the oil floating were all multiples of a single fundamental value.

The Millikan experiment is a beautiful one and the details are familiar to generations of physics students. And yet what the students are not always told is that Millikan got the answer wrong. Not very wrong, but by miscalculating the viscosity of air slightly he underestimated the charge on the electron by a factor of about one part in 150 . 
This in itself is not that interesting. Scientists get the answer wrong all the time. Even well-designed experiments can be subject to experimental error. And this was a lovely experiment that, when done perfectly, gives a perfect result. No, what was interesting was what happened next.

In the years that followed Millikan's initial experiment, others used identical apparatuses to confirm his results. And because the air-viscosity error was not generally repeated everyone started getting answers that 'disagreed' with those obtained by the great man. But the odd thing was that the results that most disagreed with the original finding (actually the ones we now know to be closest to being correct) were disregarded.

Instead, the value of the charge of the electron came to be slowly revised upwards, as if science were acting as an embarrassed student unwilling to challenge the utterances of the great professor in front of him and breaking the bad news only in tiny steps.

Feynman called things that looked like science but are not, 'Cargo Cult Science', after the cults that emerged on some South Pacific islands after the Second World War. The islanders had watched planes coming into land, and noticed that this was often accompanied by the appearance of valuable commodities from the outside world. So they built runways, control towers even, out of sticks and stones.

But of course no planes came into land. Homeopathy and crystal healing are clear examples of cargo cult science, as is the refusal to accept results that are 'wrong' only insofar as they disagree with those obtained by an eminence. We all have a long list of 'disciplines' that we can happily and safely consign to the basket labelled 'fruitloopery'. UFOs and alien kidnappings. Divining. Talking to the dead and so forth. But 'proper science' is littered with aeroplanes made of sticks and stones as well, and we must be on guard.

Talking of aeroplanes. Schoolchildren (and, more worryingly, pilots) are told how they are able to fly. They are shown a diagram, a cross section of a typical aircraft wing, with a strongly curved convex top surface and a flat or concave lower surface. They are shown the air stream, being forced to divide at the leading edge of the moving wing. They are told that because the travel path of the air over the top surface of the wing is longer than that of the air travelling over the lower surface these (top) air molecules must go faster to 'catch up' with their brethren and reunite at the trailing edge.

Back in the eighteenth century, Bernoulli showed that faster moving fluids exert a lower pressure than slow-moving equivalents, this explains why planes fly. The wing creates a partial vacuum above the wing, allowing atmospheric pressure to lift the wing upwards together with the rest of the aircraft.

This is an elegant, beautiful and simple explanation of how a plane flies. It is also, mostly, wrong - or at least it is far from being the whole story. I claim no special insights at all, but I clearly remember thinking, in school, when we were told about the Bernoulli Effect, OK then, how come planes can fly upside-down? If the cross-sectional curve of the wing is all-important this should be impossible, and yet all sorts of planes, and not just specialised stunt planes, clearly can fly upside down with a skilled pilot at the helm. Moreover, how come the model balsa-wood planes which I built, many of which had completely flat wings, were also capable of flight?

It turns out that the way planes fly is not really well understood at all and that the explanation above (which is, remember, taught in pilot school) is mostly bunkum. Why 
should the air molecules at the top of the wing rush to reunite with their lower friends? Why should they care? It turns out they don't. In fact there is a name for this wrongness, it is called the equal-transit fallacy.

So how do planes fly? It turns out that for the most part a plane wing can be more or less any shape you choose. It is its angle of attack that matters more than its crosssectional shape. Angle a flat sheet into a moving air stream and some mass of air will be deflected downwards, forcing the sheet upwards as a simple consequence of Newtonian equal-and-opposite-reaction physics. Plane wings it seems are just kites where an engine is substituted for string and wind.

Actually, it is a bit more complicated than that. The cross-section of the wing has fundamental effects on how the wing behaves at different speeds. But the message is simple. Sometimes scientific facts take on talismanic qualities that could do with some scrutiny at repeated intervals to keep everyone on the straight and narrow.

Science takes a lot of things for granted. Feynman pointed out that all too often scientist A is willing to use the control group results from an earlier experiment, performed by scientist B, in order to save time, effort and money. This can have deleterious results, as Feynman noted in his address:

Other kinds of errors are more characteristic of poor science. When I was at Cornell, I often talked to the people in the psychology department. One of the students told me she wanted to do an experiment that went something like this - it had been found by others that under certain circumstances, $\mathrm{X}$, rats did something, A. She was curious as to whether, if she changed the circumstances to Y, they would still do A. So her proposal was to do the experiment under circumstances $\mathrm{Y}$ and see if they still did A.

I explained to her that it was necessary first to repeat in her laboratory the experiment of the other person - to do it under condition $X$ to see if she could also get result $A$, and then change to $\mathrm{Y}$ and see if $\mathrm{A}$ changed. Then she would know that the real difference was the thing she thought she had under control.

She was very delighted with this new idea, and went to her professor. And his reply was, no, you cannot do that, because the experiment has already been done and you would be wasting time.

This was in about 1947 or so, and it seems to have been the general policy then to not try to repeat psychological experiments, but only to change the conditions and see what happens.

Nowadays there's a certain danger of the same thing happening, even in the famous field of physics. I was shocked to hear of an experiment done at the big accelerator at the National Accelerator Laboratory, where a person used deuterium. In order to compare his heavy hydrogen results to what might happen with light hydrogen he had to use data from someone else's experiment on light hydrogen, which was done on different apparatus. When asked why, he said it was because he couldn't get time on the program (because there's so little time and it's such expensive apparatus) to do the experiment with light hydrogen on this apparatus because there wouldn't be any new result. And so the men in charge of programs at NAL are so anxious for new results, in order to get more money to keep the thing going for public relations purposes, they are destroying possibly - the value of the experiments themselves, which is the whole purpose of the thing. It is often hard for the experimenters there to complete their work as their scientific integrity demands.

All experiments in psychology are not of this type, however. For example, there have been many experiments running rats through all kinds of mazes, and so on - with little 
clear result. But in 1937 a man named Young did a very interesting one. He had a long corridor with doors all along one side where the rats came in, and doors along the other side where the food was. He wanted to see if he could train the rats to go in at the third door down from wherever he started them off. No. The rats went immediately to the door where the food had been the time before.

The question was, how did the rats know, because the corridor was so beautifully built and so uniform, that this was the same door as before? Obviously there was something about the door that was different from the other doors. So he painted the doors very carefully, arranging the textures on the faces of the doors exactly the same. Still the rats could tell. Then he thought maybe the rats were smelling the food, so he used chemicals to change the smell after each run. Still the rats could tell. Then he realized the rats might be able to tell by seeing the lights and the arrangement in the laboratory like any commonsense person. So he covered the corridor, and still the rats could tell.

He finally found that they could tell by the way the floor sounded when they ran over it. And he could only fix that by putting his corridor in sand. So he covered one after another of all possible clues and finally was able to fool the rats so that they had to learn to go in the third door. If he relaxed any of his conditions, the rats could tell.

Now, from a scientific standpoint, that is an A-number-one experiment. That is the experiment that makes rat-running experiments sensible, because it uncovers the clues that the rat is really using - not what you think it's using. And that is the experiment that tells exactly what conditions you have to use in order to be careful and control everything in an experiment with rat-running.

I looked into the subsequent history of this research. The next experiment, and the one after that, never referred to Mr. Young.

They never used any of his criteria of putting the corridor on sand, or being very careful. They just went right on running rats in the same old way, and paid no attention to the great discoveries of Mr. Young, and his papers are not referred to, because he didn't discover anything about the rats. In fact, he discovered all the things you have to do to discover something about rats. But not paying attention to experiments like that is a characteristic of cargo cult science.

Some claim that the infection of cargo cultism runs deep throughout science. In 2011 the New Yorker magazine published a piece by the journalist Jonah Lehrer called The Truth Wears Off. Lehrer's article was basically saying that science is broken. His arguments are well-expressed, provocative and, initially at least, rather convincing.

He uses several examples to show that there is something rotten at the heart of the scientific state. The focus of the argument is something called the Decline Effect, the name given to the odd phenomenon whereby scientific claims receive decreasing support over time.

For instance, a study may show that a particular drug is strongly efficacious at treating, say, depressive illness. The drug becomes the new standard for treatment, but then something strange happens. After time, the potency of the drug declines. Follow-up trials show less and less effect and eventually, in the case he quotes, a whole series of anti-psychotic and anti-depressive medicines considered state of the art when they were developed in the 1990s are now thought to be no more effective than first-generation treatments developed in the 1950s.

Does Lehrer have a point? There is an obvious objection to this thesis which says that all we are seeing is simple regression to the mean. Of course, spectacular results are unlikely to be repeated, at least when you are measuring something that can be on an 
almost infinitely variable scale (such as the efficacy of a new drug to treat a mental disease) rather than something fixed (such as the mass of the Higgs Boson). There is nothing spooky going on. The Universe is not doing its best to stop us understanding how it works. We do not see the decline effect in physics, for example, or cosmology. The cosmic microwave background does not change every time we measure it. Nor does $\mathrm{G}$ or any other of the physical constants. Drugs that were effective against major diseases 30 years ago remain effective today, and if they do not the reasons why (such as the over prescription of antibiotics) are well understood. Science is real.

But Lehrer makes the point that science needs to be on its guard. We have to be careful and watch out not only for fraud but for myth and complacency. We need to be aware, for example, that 'replicability', a cornerstone of the scientific method, does not necessarily mean that even ground-breaking studies are actually replicated. It came as a surprise to me to discover how few experiments published in the journals are indeed repeated by other scientists to see if they get the same results. I would guess that the more distinguished the researcher who made the original finding the less likely this is to happen. Science really does progress one funeral at a time.

We must be careful to watch out for cherry-picking and publication bias, the rejection of findings that find nothing. In the 1990s, a slew of (mostly American) studies were published purporting to show the effects of 'intercessory prayer', the idea that praying for the sick has a positive effect on their recovery. It turns out that a great deal of cherry picking was going on and there is (as we would expect in a sane universe) no deity up there who decides to kill some people and not others depending on whether a third party is asking Him/Her to do so.

We must be on guard against pseudoscience but be careful not to throw the baby out with the bathwater. It was quite wrong, for instance, as some sceptics attest, that if a small but real effect could be discovered in the field of parapsychology, perhaps the discovery of a limited telepathic ability in a tiny subset of the population, then this would be of little importance. Quite the opposite. The confirmed existence of telepathy would shake science to its foundations. We can be sceptical about UFOs but not too sceptical about alien life in general.

And we must be careful not to cherish neat theories just because they are old and held by everyone and taught in schools. The more we learn about the relationship between DNA, RNA, genes and proteins the more complex the story becomes. Most physicists accept that string theory is the way forward in the quest to unite the world of the quantum with gravity, but a minority of physicists point out that this is the case largely because it is hard to get a research grant if you do not believe in string theory.

Sometimes science works out just as it should. Sometimes it surprises us. And sometimes it just goes horribly wrong. Success or failure, we should treat those impostors just the same. And take absolutely no one's word for it.

\section{About the Author}

Michael Hanlon is a freelance science journalist and author. 